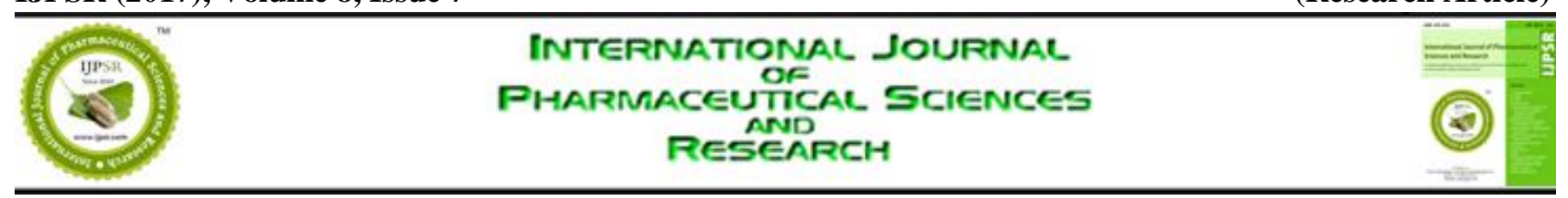

Received on 03 January, 2017; received in revised form, 14 February, 2017; accepted, 24 February, 2017; published 01 July, 2017

\title{
PREVALENCE OF URINARY TRACT INFECTION IN CHILDREN WITH NEPHROTIC SYNDROME
}

Morteza Salarzaei ${ }^{1}$, Soleyman Saravani ${ }^{2}$, Mehdi Heydari ${ }^{1}$, Halime Aali ${ }^{3}$, Alireza Malekzadegan ${ }^{4}$, Dadkhoda Soofi ${ }^{3}$, Ehsanollah Rahimi Movaghar ${ }^{4}$, Shahram Mir ${ }^{5}$ and Fateme Parooei ${ }^{* 1}$

Students Research Committee ${ }^{1}$, Department of Preventive Medicine And Social Medicine ${ }^{2}$, Department of Internal Medicine ${ }^{3}$, Amir Al-Momenin Hospital, Department of Surgery ${ }^{4}$, Student Laboratory Science 5, Students Research Committee, Zabol University of Medical Science, Zabol, Iran.

Keywords:

Urinary tract infection, Nephrotic Syndrome, Children

Correspondence to Author:

Fateme Parooei

Medical student

Students Research Committee,

Zabol University Of Medical

Science, Zabol, Iran.

E-mail: fatemeparooie@gmail.com

\begin{abstract}
Introduction: Nephrotic syndrome is the most common chronic glomerular diseases in children. The most common infection in children with nephrotic syndrome is urinary tract infection. The aim of this study was to evaluate the prevalence of urinary tract infection in children with nephrotic syndrome and to prepare and submit an appropriate protocol regarding the necessity and method of UTI examination on these children. Materials and Methods: The population in this descriptive study included children aged six months to 14 years old with nephrotic syndrome referred to pediatric ward in Imam Ali (AS) Hospital in Zahedan in Iran during 2015. To collect and record the required data, a questionnaire was used that included questions about the past history, reason for referral, and medical tests of the patients. The collected data were entered into SPSS software. Results: The subjects were 124 children with nephrotic syndrome ( 71 males and 53 females) with a mean age of 6.213 .1 years (at least 6 months and at most 14 years) were examined. Prevalence of infection in study samples was estimated $38 \%(n=47)$. In $69.2 \%$ of the cases that kidney echo was high, the children had infection. The results of this study showed that the most common microorganisms involved in urinary tract infection were: Escherichia coli (13\%), Klebsiella (13\%), Enterococcus (6\%), Proteus (4\%) and other microorganisms (4\%). Conclusion: When a child is diagnosed with nephrotic syndrome, it is necessary to examine the disease so as to discover and prevent the risk of potential complications specifically urinary tract infection by initiating appropriate therapy.
\end{abstract}

INTRODUCTION: Nephrotic syndrome is the most common chronic glomerular diseases in children. The incidence of this syndrome in children is $2-7$ cases per $100.0000^{1}$. Most cases of nephrotic syndrome $(90 \%)$ are the idiopathic type. The remaining $10 \%$ have a secondary idiopathic nephrotic syndrome to glomerular disease or systemic diseases ${ }^{2}$.

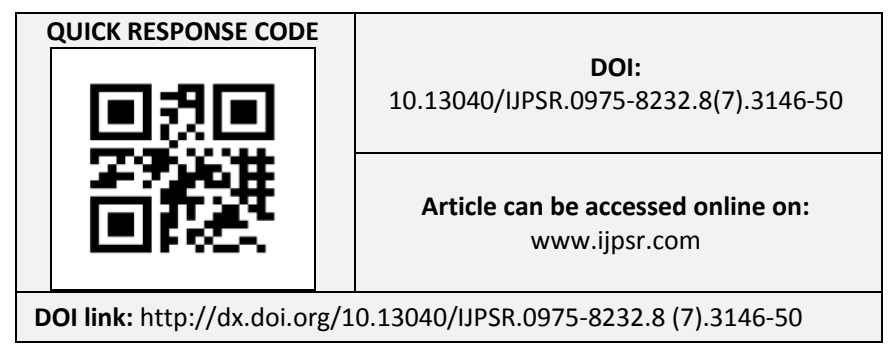

Nephrotic syndrome may be primary (including disease with minor changes and focal segmental glomerulosclerosis) or secondary to a systemic disease like lupus erythematosus ${ }^{3}$. Patients with nephrotic syndrome suffer different complications including infection, hypovolemia, hyperlipidemia, effusion and hyper coagulability ${ }^{4}$. When children with nephrotic syndrome enter pediatric ward in the hospital, it is necessary to examine them at first to avoid potential complications or infections are discovered immediately and appropriate therapy is initiated ${ }^{5}$. Nephrotic syndrome increases a child's susceptibility to infection ${ }^{6}$. IgG (a type of protein) is lost in the urine, so the body's ability to fight against microorganisms is reduced and the child becomes susceptible to infections because of 
immune suppression ${ }^{7}$. The most common infection in children with nephrotic syndrome who are diagnosed with a urinary tract infection is urinary tract infection (UTI) ${ }^{8}$. Urinary tract infection is the second most common bacterial disease in children after upper respiratory tract infection and it is the most common bacterial infection that affects humans throughout life ${ }^{9}$. Symptoms of urinary tract infection are different regarding age and the younger a child is, the more non-specific are these symptoms ${ }^{10}$. Due to fact that the lack of early detection of urinary tract infection can cause kidney scar and increased blood pressure and the progression of nephrotic syndrome as the most common chronic kidney disease in children, it is necessary to consider the diagnosis of nephrotic syndrome in any child with the disease. The aim of this study was therefore to evaluate the prevalence of urinary tract infection in children with nephrotic syndrome and to prepare and submit an appropriate protocol regarding the necessity and method of UTI examination on these children.

MATERIALS AND METHODS: The study population in this descriptive study included children aged six months to 14 years old with nephrotic syndrome referred to pediatric ward in Imam Ali (AS) Hospital in Zahedan in Iran in 2015. Urine specimen (after washing the perineum) in children who had bladder control were collected using urine midstream and in children without bladder control, the specimen were preferably collected using suprapubic or catheter method and in the event of failure, the specimen were collected from urine bag and were immediately sent to the laboratory to perform the complete urinalysis and urine culture. If urinalysis report includes positive pyuria or nitrite, the early test is considered active and when the number of microorganisms of a species in the specimen of urine midstream is more than $10^{4}$, urine cultures is considered positive and in case the catheter specimen microorganisms are more than $10^{3}$, any bacterial growth in culture as well as suprapubic samples are considered positive. Obviously, the diagnosis of urinary tract infection in children is confirmed when urinalysis is consistent with urine culture results. Inclusion criteria were: children six months to 14 years with nephrotic syndrome, avoiding the use of any oral antibiotics and injection 48 hours before laboratory test. Exclusion criteria were: children less than 6 months and more than 14 years and prior antibiotics consumption. To collect and record the required data, a questionnaire was used that asked about the history, reason for referral and laboratory tests. The gathered data was entered SPSS software and to describe the data, frequency tables, statistical indicators and diagrams were used. To analyze the relationships between variables, related tests including the chi-square test 2, t-test and ANOVA were used.

RESULTS: 124 children with nephrotic syndrome (71 males and 53 females) with a mean age of $6.21 \pm 3.1$ years (at least 6 months and at most 14 years) were examined. Prevalence of infection in study samples was estimated $38 \% \quad(n=47)$. The average age of children without infection was $6.57 \pm 3.18$ years and the infected group age was $5.48 \pm 3.36$ years. According to Table 1 no significant difference was observed considering white blood count, hemoglobin level, erythrocyte sedimentation rate (ESR), the amount of creatinine $(\mathrm{Cr})$, nitrogen, BUN, serum total protein and urinary protein.

TABLE 1: THE PARAMETERS IN PATIENTS WITH NEPHROTIC SYNDROME

\begin{tabular}{|c|c|c|c|}
\hline Chemical parameters - infection & Negative & Positive cases & $\mathbf{P}$ \\
\hline Serum albumin $(\mathrm{g} / \mathrm{dl})$ & $2.61 \pm 0.61$ & $85 \% \pm 2.55$ & $<2 \%$ \\
\hline Serum total protein (g/day) & $4.65 \pm 0.67$ & $4.65 \pm 0.67$ & $<0.16$ \\
\hline Urinary protein excretion (g/day) & $4955 \pm 4860.4$ & $4241 \pm 3962$ & $<0.16$ \\
\hline The number of white blood cells & $10856 \pm 4760$ & $9942 \pm 2861$ & $<0.37$ \\
\hline Hemoglobin $(\mathrm{g} / \mathrm{dl})$ & $12.3 \pm 1.98$ & $12.8 \pm 1.91$ & $<0.16$ \\
\hline Erythrocyte sedimentation rate $(\mathrm{mm} / \mathrm{h})$ & $66 \pm 40$ & $74 \pm 36$ & $<0.43$ \\
\hline Blood creatinine $(\mathrm{mg} / \mathrm{dl})$ & $76 \% \pm 43 \%$ & $62 \% \pm 0.21$ & $<0.18$ \\
\hline Nitrogen, blood urea (mg/ dl) & $16.57 \pm 9.38$ & $17.8 \pm 8.58$ & $<0.44$ \\
\hline Serum triglycerides (mg/dl) & $436 \pm 201$ & $335 \pm 195$ & $<0.14$ \\
\hline Serum Cholesterol (mg/dl) & $456 \pm 171$ & $373 \pm 146$ & $<0.007$ \\
\hline
\end{tabular}


Regarding the kidney echo the patients were high echo). In $69.2 \%$ of the cases that the kidney divided into three groups: (normal echo, low echo, echo was high, there was infection.

TABLE 2: FREQUENCY DISTRIBUTION OF PATIENTS WITH THE NEPHROTIC SYNDROME ACCORDING TO THE RESULTS OF RENAL BIOPSY WITH NEGATIVE AND POSITIVE INFECTION RESULTS

\begin{tabular}{|c|c|c|c|}
\hline Kidney biopsy results - infection & $\begin{array}{c}\text { Positive } \\
\mathrm{n}=47\end{array}$ & $\begin{array}{c}\text { Negative } \\
\mathrm{n}=77\end{array}$ & $\begin{array}{c}\text { Total } \\
124\end{array}$ \\
\hline Nephrotic syndrome with mild scar (mcws) & 20 & 40 & 60 \\
\hline Mesangioproliferative (mespgn) & 15 & 17 & 32 \\
\hline Membranoproliferative & 4 & 6 & 10 \\
\hline Congenital nephrotic syndrome (CNS) & 4 & 3 & 7 \\
\hline Focal segmental glomerulosclerosis (FSGS) & 0 & 7 & 7 \\
\hline Membranous (MGN) & 2 & 3 & 5 \\
\hline Amyloidosis & 2 & 1 & 3 \\
\hline
\end{tabular}

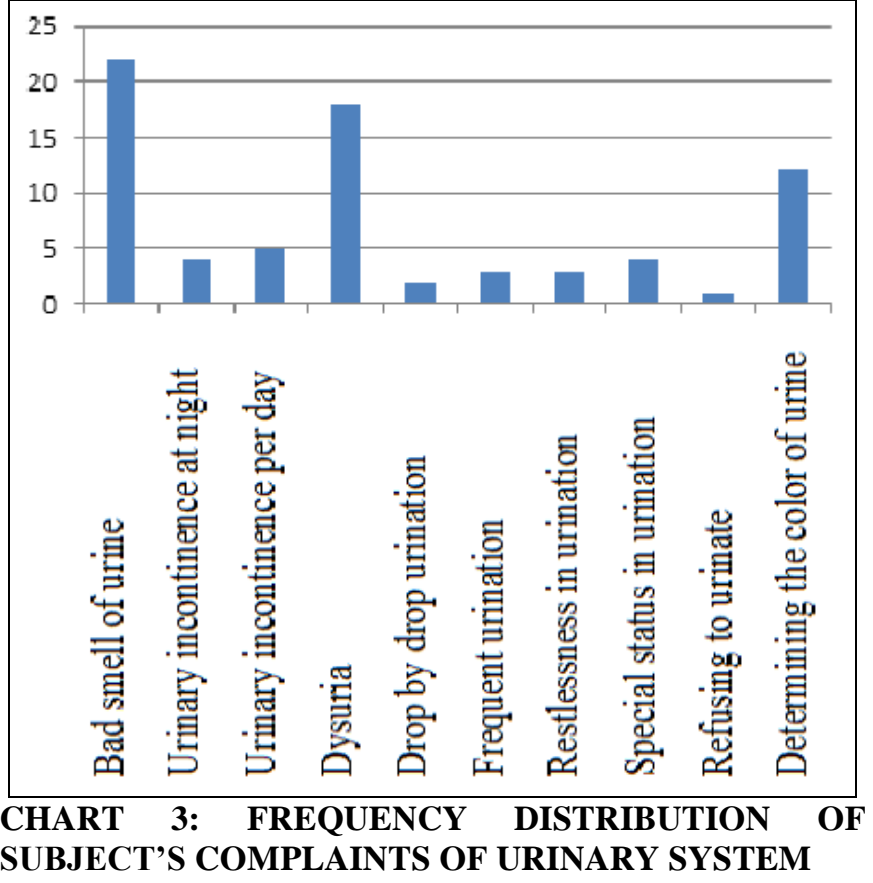

The results of this study showed that the most common microorganisms involved in urinary tract infection are: Escherichia coli (13\%), Klebsiella (13\%), Enterococcus (6\%), Proteus (4\%) and other microorganisms (4\%).

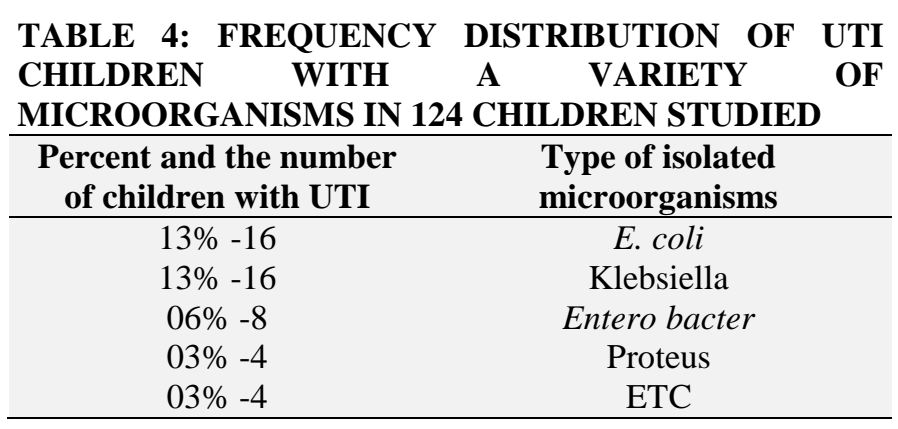

As the table shows, most bacteria involved in urinary tract infections are: Klebsiella and E. coli, each with 13 percent. The results showed that most children with UTI aged more than 2 years old.
TABLE 5: FREQUENCY DISTRIBUTION OF 124 CHILDREN (BOYS AND GIRLS) OF DIFFERENT AGES WITH UTI IN THE STUDY

\begin{tabular}{ccc}
\hline & \multicolumn{2}{c}{ Percent of infected children } \\
\cline { 2 - 3 } Age & female & Male \\
\hline 6-12 months & 4 & 3 \\
1-2 years & 4 & 6 \\
2-8 & 7 & 5 \\
$8-14$ & 3 & 6 \\
\hline
\end{tabular}

As the table shows, most children with UTI in both sexes age above two years old.

TABLE 6: CLINICAL SYMPTOMS OF CHILDREN WITH URINARY TRACT INFECTION.

\begin{tabular}{ccc}
\hline Signs & Number & Percent \\
\hline Fever & 24 & 50 \\
Seizures due to fever & 11 & 23 \\
Seizures caused by meningitis & 7 & 6 \\
Diarrhea & 25 & 20 \\
Vomiting associated with diarrhea & 20 & 16 \\
Vomiting associated with meningitis & 7 & 6 \\
Lethargy & 7 & 6 \\
Coma & 4 & 3 \\
Respiratory symptoms & 20 & 16 \\
Jaundice & 16 & 13 \\
Urinary retention & 4 & 3 \\
Hepatoslenomegaly & 7 & 6 \\
Bad smell of urine & 22 & 18 \\
\hline
\end{tabular}

As it is clear, the most observed sign in pediatric urinary tract infections are associated with fever and the least observed sign are related to urinary retention.

DISCUSSION: In the age group 5.32 \pm 3.12 years with the female gender that referred to the hospital with nephrotic syndrome, urinary tract infection was relatively more common. In a study by Derakhhan et al., in Iran, the medical records of all children aged 1 to 10 years old with a clinical diagnosis of nephrotic syndrome who had at least 7 years of regular visits to the clinic, the age of the outbreak, sex, number of relapses, infection and 
damage were evaluated. Their findings showed that in the age group of $5.82 \pm 2.24$ years, urinary tract infection in females was more common ${ }^{11}$. This reflects the impact of racial factors in the gender distribution of the disease. The recurrent urinary tract infection in the age group of 6 months to 8 years was significantly lower than the group aged $8-14$ years $(\mathrm{P}=0.001)$. This could be indicative of the fact that structural abnormalities of the kidneys and the bladder in patients older than 8 years are more stable and cause recurrent urinary tract infections. In this study, $15 \%$ of the children aged 6 months to 8 years and $17 \%$ of children aged 8-14 years had abnormal ultrasound that the statistical difference was significant $(\mathrm{P}=0.005)$.

This may have increased the incidence of recurrence urinary tract infection in children above 8 years. Therefore, urinalysis and urine culture should be considered in children with nephrotic syndrome and with early diagnosis of urinary tract infection, required follow up including isotope scan (DMSA) cryptography during urination (VCUG) and ultrasound could be performed to prevent the adverse effects. Most of the symptoms observed in urinary tract infection in children were related to fever. Febrile seizures were the most common disorder observed in children with urinary tract infection in this study; although generally with poor prognosis, it can represent a serious infection with bacterial background. Therefore, each child with seizures associated with fever should be carefully examined and evaluated in detail to determine the cause of the fever.

The incidence of urinary tract infection in young children is of particular importance because UTI in this age group may not produce obvious clinical symptoms other than fever and if it is not diagnosed, it may cause damage to the kidney (scar) in patients. In addition, in this age group urine samples are not prepared as easily as the older patients and sometimes it is needed to take the sample using catheterization or suprapubic aspiration through the urethra that will not be easily accepted by children and parents. In a study released in 2016 by Trainor, urinary tract infection report was $5.9^{12}$. In this study, children with febrile seizure caused by a urinary tract infection were significantly associated with gender (female) $(\mathrm{P}=0.001)$. This is similar to the results of the present study, but the number of subjects in the study by Trainor was 170 people.

Levine conducted a study in 2014 and the incidence of febrile seizures in children with urinary tract infection in his report was $90 \%{ }^{13}$. In this study, similar results were not observed; that is of 30 children with urinary tract infection, 15 patients which means $50 \%$ had fever.

The most common complaint in children's urine was bad smell of urine with 0.18. Specific symptoms such as urinary tract infection, dysuria, dribbling urination, frequent urination, restless urination and refuse to urinate were observed in a relatively small number of children (respectively $18 \%, 02 \%, 03 \%, 03 \%$ and $01 \%$ ). The review study conducted in 2015 by Shiekh et al., reported the prevalence of complaints of patients as: fever, suprapubic pain, abdominal pain, back pain, dysuria, urinary frequency and urinary incontinence 14.

In this study, the clinical signs of kidney infection are: fever and chills, back pain, vomiting, restlessness, loss of appetite, decreased urine volume and clinical signs of dehydration. E. coli outbreak with $13 \%$, was reported the most common urinary germ and in the next ranks were Klebsiella $(13 \%)$ and Entero bacter (06\%) that were similar to most other studies ${ }^{15-17}$. 32\% of children had abnormal ultrasound. Edler et al., studied the prevalence of abnormal ultrasound and the result was $30 \%$. In the study by Oyetunj et al., the abnormality was reported $14.818,19$. The higher incidence of renal failure in the present study could be because this study was conducted in children with nephrotic syndrome and it is natural that the possibility of structural problems in these patients is higher.

In addition, because ultrasound is operator dependent and in this study ultrasound was performed by an experienced sonographer in children, it seems that the diagnosis of renal failure has been more careful.

CONCLUSION: When a child is diagnosed with nephrotic syndrome, it is necessary to examine them to prevent or quickly discover potential complications specifically urinary tract infection and to initiate proper therapy. 
ACKNOWLEDGMENT: The authors would like to acknowledge the Zahedanl University of Medical Sciences research and health assistants for their cooperation during the study.

\section{REFERENCES:}

1. Kamei K, Takahashi M, Fuyama M, Saida K, Machida H and Sato M: Rituximab-associated agranulocytosis in children with refractory idiopathic nephrotic syndrome: case series and review of literature. Nephrology Dialysis Transplantation. 2015; 30(1): 91- 6

2. Hahn D, Hodson EM, Willis NS and Craig JC: Corticosteroid therapy for nephrotic syndrome in children. The Cochrane Library. 2015.

3. Pittet LF, Posfay-Barbe KM, Chehade H, Rudin C, Wilhelm-Bals $\mathrm{A}$ and Rodriguez $\mathrm{M}$ : Optimizing seroprotection against pneumococcus in children with nephrotic syndrome using the 13-valent pneumococcal conjugate vaccine. Vaccine. 2016; 34(41): 4948-54.

4. Sinha A, Bajpai J, Saini S, Bhatia D, Gupta A and Puraswani M: Serum-soluble urokinase receptor levels do not distinguish focal segmental glomerulo sclerosis from other causes of nephrotic syndrome in children. Kidney International. 2014; 85(3): 649-58.

5. Sreenivasa B, Murthy CS, Raghavendra K, Basavanthappa S, Pejaver R and Jadala HV: Urinary tract infection at presentation of nephrotic syndrome: A clinical evaluation. Indian Journal of Child Health. 2015; 2(1): 1-4.

6. Wyatt RJ and Julian BA. IgA nephropathy. New England Journal of Medicine. 2013; 368(25): 2402-14.

7. Ajayan P, Krishnamurthy S, Biswal $\mathrm{N}$ and Mandal J: Clinical spectrum and predictive risk factors of major infections in hospitalized children with nephrotic syndrome. Indian pediatrics. 2013; 50(8): 779-81.

8. Pravitsitthikul N, Willis NS, Hodson EM and Craig JC: Non-corticosteroid immunosuppressive medications for steroid-sensitive nephrotic syndrome in children. The Cochrane Library. 2013.

9. Morris BJ and Wiswell TE: Circumcision and lifetime risk of urinary tract infection: a systematic review and metaanalysis. The Journal of Urology. 2013; 189(6): 2118-24.

10. Stein R, Dogan HS, Hoebeke P, Kočvara R, Nijman RJ and Radmayr C: Urinary tract infections in children: EAU
/ ESPU guidelines. European urology. 2015; 67(3): 54658.

11. Derakhshan A, Derakhshan D, Fallahzadeh $\mathrm{MH}$ and Basiratnia M: Recurrence of nephrotic syndrome following kidney transplantation in a child with congenital nephrotic syndrome. Saudi Journal of Kidney Diseases and Transplantation. 2016; 27(1): 150.

12. Trainor-Moss $\mathrm{S}$ and Mutapi F: Schistosomiasis therapeutics: what's in the pipeline? Expert review of clinical pharmacology. 2016; 9(2): 157-60.

13. Levine A, Koletzko S, Turner D, Escher JC, Cucchiara S and De Ridder L: ESPGHAN revised porto criteria for the diagnosis of inflammatory bowel disease in children and adolescents. Journal of pediatric gastroenterology and nutrition. 2014; 58(6): 795-806.

14. El-Sheikh AA, El-Sheikh NA, Badawi MM, Mahran MH and Khalaf NA. Diabetic Autonomic Neuropathy, a Cofactor related to Recurrent Urinary Tract Infections In Type 2 Diabetic females, a Study in an Egyptian Population. Journal of American Science. 2015; 11(2s).

15. Ramadan DS, Bassyoni EA, Amer MM and Emam SM: Detection of ESBL Producing Bacteria in Cases of Urinary Tract Infection in Pediatric Department at Benha University Hospital. The Egyptian Journal of Medical Microbiology (EJMM). 2016; 25(1).

16. Fan NC, Chen HH, Chen CL, Ou LS, Lin TY and Tsai $\mathrm{MH}$ : Rise of community-onset urinary tract infection caused by extended-spectrum $\beta$-lactamase-producing Escherichia coli in children. Journal of Microbiology, Immunology and Infection. 2014; 47(5): 399-405.

17. Gurevich E, Tchernin D, Schreyber R, Muller R and Leibovitz E: Follow-up after infants younger than 2 months of age with urinary tract infection in Southern Israel: epidemiologic, microbiologic and disease recurrence characteristics. Brazilian Journal of Infectious Diseases. 2016; 20(1): 19-25.

18. Elder JS and Diaz M: Vesicoureteral reflux-the role of bladder and bowel dysfunction. Nature Reviews Urology. 2013; 10(11): 640-8.

19. Oyetunji TA, Ong'uti SK, Bolorunduro OB, Gonzalez DO, Cornwell EE and Haider AH: Epidemiologic trend in elderly domestic injury. Journal of surgical research. 2012; 173(2): 206-11.

How to cite this article:

Salarzaei M, Saravani S, Heydari M, Aali H, Malekzadegan A, Soofi D, Movaghar ER, Mir S and Parooei F: Prevalence of urinary tract infection in children with nephrotic syndrome. Int J Pharm Sci Res 2017; 8(7): 3146-50.doi: 10.13040/IJPSR.0975-8232.8(7).3146-50.

All @ 2013 are reserved by International Journal of Pharmaceutical Sciences and Research. This Journal licensed under a Creative Commons Attribution-NonCommercial-ShareAlike 3.0 Unported License.

This article can be downloaded to ANDROID OS based mobile. Scan QR Code using Code/Bar Scanner from your mobile. (Scanners are available on Google Playstore) 